\title{
Overcoming Tortuous Anatomy in Intracranial Intervention
}

\author{
Mathew Cherian ${ }^{1}$ Pankaj Mehta ${ }^{1} \quad$ Poyyamoli Santhosh $^{1} \quad$ Kareparambil R. Rahul $^{1}$ Gandhi Jenny ${ }^{1}$ \\ Swamiappan Elango ${ }^{1}$
}

${ }^{1}$ Department of Interventional Radiology, Kovai Medical Center and

Hospital, Coimbatore, Tamilnadu, India

\begin{abstract}
Address for correspondence Mathew Cherian, Department of Radiology, Kovai Medical Center and Hospital, Coimbatore, Tamilnadu, India (e-mail: dr.mathewcherian@gmail.com).
\end{abstract}

J Clin Interv Radiol ISVIR 2018;1:38-41

\begin{abstract}
Keywords

- tortuous vessels

- neuro intervention

- difficult access

Intracranial interventions comprise a set of procedures, which are complex and challenging. Further, they are extremely risky because complications in the brain are usually associated with significant mortality and morbidity. Thus, a clear understanding is necessary to handle tortuous vessels because improper planning can result in a higher rate of complications. The combination of long sheaths, specialty wires, and preshaped catheters can enable us to access some of the most complex vascular anatomy. There are several conditions that can contribute to a difficult access. There are few techniques described by different authors. The authors have listed the techniques found useful based on their experience. These challenges include tortuous iliac arteries, dilated tortuous aortic arch, acute takeoff of the arch vessels, tortuous neck vessels with prominent loops, crossing a large aneurysm, extensive peripheral vascular disease, etc.
\end{abstract}

\section{Introduction}

The field of neurointervention has grown enormously over the last two decades. We have better microcatheters, coils, balloons, and wires than ever before. However, none of these devices have any use until we are able to reach the target site. Often, complex vascular anatomy can pose a problem to navigate microcatheters and balloons to the actual site of pathology. This article attempts to help a beginner overcome some of the challenges one can face during endovascular treatment of vascular pathology in a patient with tortuous vascular anatomy. ${ }^{1-3}$

\section{Tortuous Iliac Arteries}

Vessel tortuosity results in lack of pushability of catheters and also the loss of torque on the guidewires that are necessary to cannulate vessels. Further, tortuous vessels can prevent transmission of energy over the catheter, and these catheters may suddenly move forward resulting in a dissection or perforation. This can result in longer procedure times and thus complications.
The simplest way to cross a tortuous iliac artery is to use a combination of a simple angled catheter (Berenstein's) along with a Terumo angled Glidewire ( $\boldsymbol{- F i g}$. 1). These are nitinol wires, which have a good 1:1 torque. Further, if the procedure is done under road map, the chances of dissection become extremely low. Once the catheter is in the aorta, it is recommended that the catheter is exchanged for a $40-$ or a $70-\mathrm{cm}$ long sheath to ensure that all the tortuosity in the iliac artery

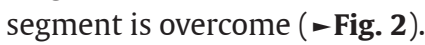

\section{Overcoming a Tortuous Dilated Arch}

Once the aorta elongates, the arch vessels become difficult to cannulate as it becomes angulated and rotates in the anterior-posterior direction. Based on this angulation, the arch is divided into three types: types 1,2 , and 3 , which is based on whether all three vessels arise at the same transverse plane at the convexity of the arch or whether the innominate and the left carotids go way below the apex of the arch. The best view to profile the arch is the left anterior oblique and one of the best catheters used to engage the vessels in an unfolded
Copyright $@ 2018$ by Indian Society of Vascular and Interventional Radiology
License terms

()(1) $\Theta \circledast$ 


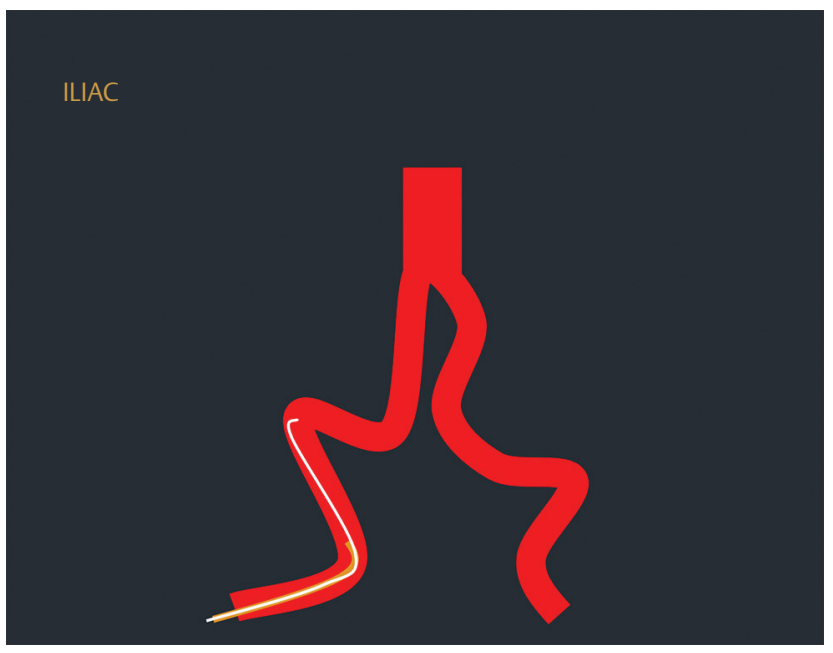

Fig. 1 Combination of a simple angle catheter and angled Glidewire helps cross-tortuous vessels.

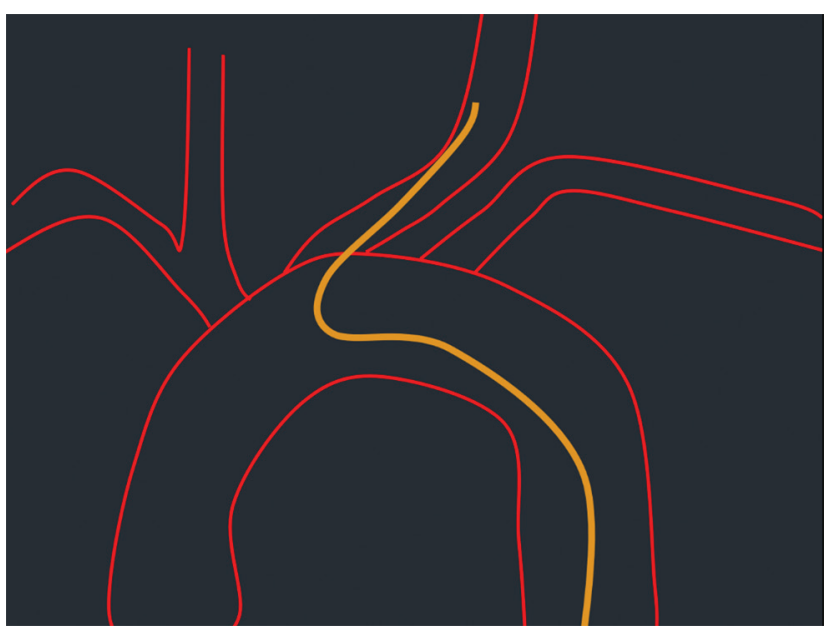

Fig. 3 Simmons catheter can be used to cannulate vessels from an unfolded arch.

arch is the Simmons 1 or 2 (-Fig. 3 ). There are many ways to make the curve in the Simmons 1 . The most common way is to first take the catheter up to the proximal descending aorta over a J wire. The wire is then pulled back by approximately $10 \mathrm{~cm}$, and the catheter tip is pushed against the outer curve of the arch to enable it to form a loop and then a clockwise rotation is given, following which a clockwise torque is given to the catheter with the guidewire in the artery in the same position. At times, the catheter forms a tighter loop rather than actually forming the Simmons 1 shape. In such a situation, the guidewire is gently pushed distally to enable the loop to open up. ${ }^{4}$ Sometimes these techniques will not work, especially when the aorta is grossly dilated and a Simmons 2 has to be used. In this situation the Simmons catheter is taken to the root of the aorta and the guidewire pulled back by $10 \mathrm{~cm}$, following which this catheter tip is pushed against the annulus to form the loop and the catheter is torqued to stabilize the shape. Once the ostium is engaged, a road map is taken and an exchange length angled Terumo wire is navigated deep into a branch of the external carotid artery. The

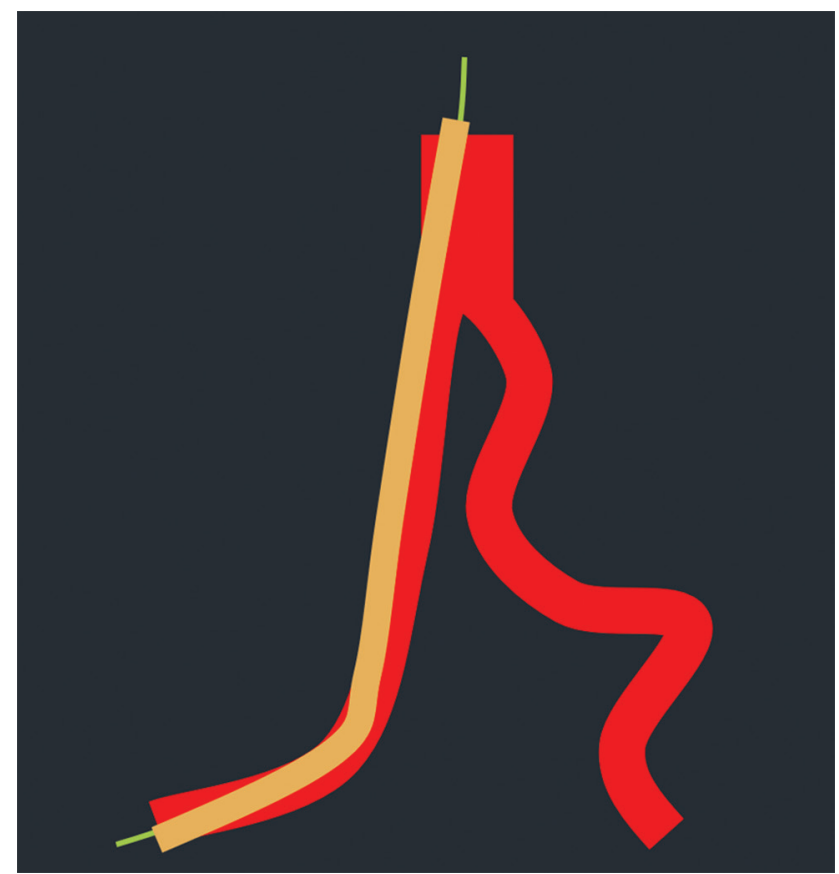

Fig. 2 A long sheath can correct tortuosity.

Simmons 1 is then exchanged for a catheter with a simple curve like the Berenstein, and the angled Terumo wire is then replaced with an exchange length Amplatz extra stiff straight wire. A long sheath or the diagnostic catheter of choice is then tracked over the extra stiff wire. The long sheath is positioned at the ostium of the common carotid artery. Once the long sheath is in position, changing of catheters become easy and it also gives stability to the guiding catheter for neurointerventions. If one has a biplane digital subtracted angiography (DSA), one can make use of the lateral plane to see the distal end of the wire and the frontal plane to see the arch by overcoming the default isocenter position and positioning the lateral plane distal to the frontal plane. Your application expert will be able to tell you how this can be achieved in your cath laboratory.

\section{Separating the Right Subclavian and the Right Carotid Artery}

The best view to separate the right carotid and the right subclavian artery is the right anterior oblique of approximately 30 degrees, following which the same technique that was used in the previous paragraph can be used (-Fig. 4).

\section{Managing Multiple Internal Carotid Artery Loops}

Once the long sheath is in position, we may often find multiple loops in the internal carotid artery making it difficult to access the intra-cranial vessels. The way to overcome this is to take an access catheter such as the Neuron (Penumbra Devices), Fargo (Balt Devices) or any other distal access catheter with a minimum internal diameter of 0.07 in. Before placing these catheters, it is mandatory that the internal carotid 


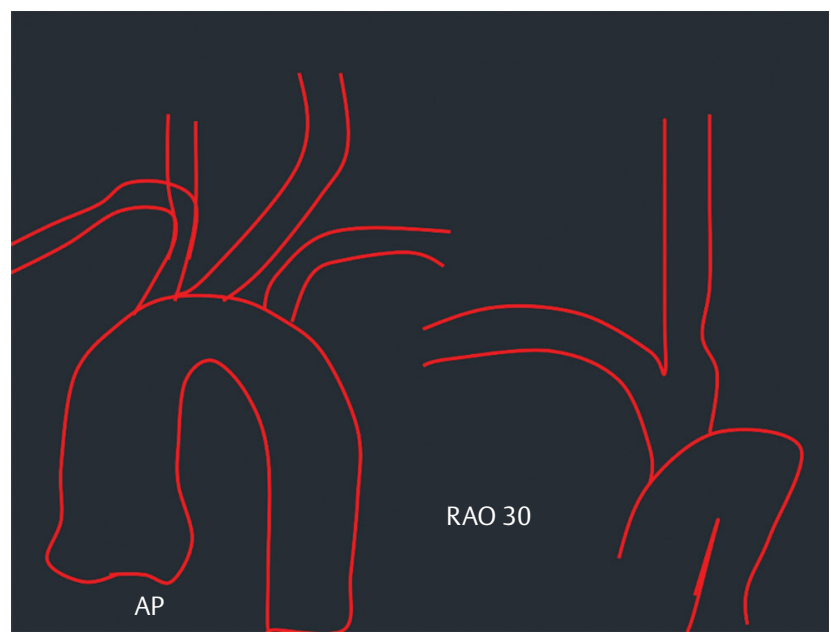

Fig. 4 Right anterior oblique (RAO) helps separate the right carotid from the right subclavian. AP, anteroposterior.

artery is primed with $1 \mathrm{mg}$ of nimodipine $(5 \mathrm{~mL})$, which is diluted in $50 \mathrm{~mL}$ of saline and injected over 10 minutes. Although intra-arterial nimodipine has been practiced in the treatment of intracranial vasospasm, ${ }^{5}$ it is mandatory that an intra-arterial pressure monitoring line is available during nimodipine infusion and the infusion is stopped when the mean arterial pressure drops below $50 \mathrm{~mm} \mathrm{Hg}$. The anesthetist is also informed before the nimodipine infusion is initiated to ensure management of drop in blood pressure (BP) during this step. The long sheath is normally kept close to the origin of the common carotid artery. Following this, the distal access catheter is then navigated over a $125-\mathrm{cm} 4 \mathrm{~F}$ catheter and Terumo Glidewire combination ( - Fig. 5). This step is important to prevent a dissection when the distal access catheter is pushed across tortuous loops. The distal access catheter is navigated to a point close to the carotid siphon. It is extremely important that the long sheath is kept in view as you push the distal access catheter because the long sheath tends to be pushed back out of the carotid artery.

\section{Direct Carotid Access}

At times, the arch may be so difficult that it is impossible to access a carotid artery. ${ }^{3}$ For example, in a patient who has got chronic type A dissection or an extremely unfolded aortic arch, the only way out is the direct carotid puncture.

The carotid artery is accessed using radial puncture or a pediatric puncture set under ultrasound guidance at the lowest possible point in the neck. A road map is then taken through the sheath or needle, and a guidewire is then navigated into the external carotid artery ( - Fig. 6). This is exchanged for a 5 or $6 \mathrm{~F}$ sheath depending on whether a single microcatheter or two devices are going to be used. The sheath is then positioned at the origin of the internal carotid artery, and the internal carotid artery is primed with nimodipine. Following this, the guiding catheter is navigated to a position, which is comfortable, and then the sheath along with the guiding catheter is either sutured to the skin or stuck using Tegaderm (3M). This step is extremely crucial because there

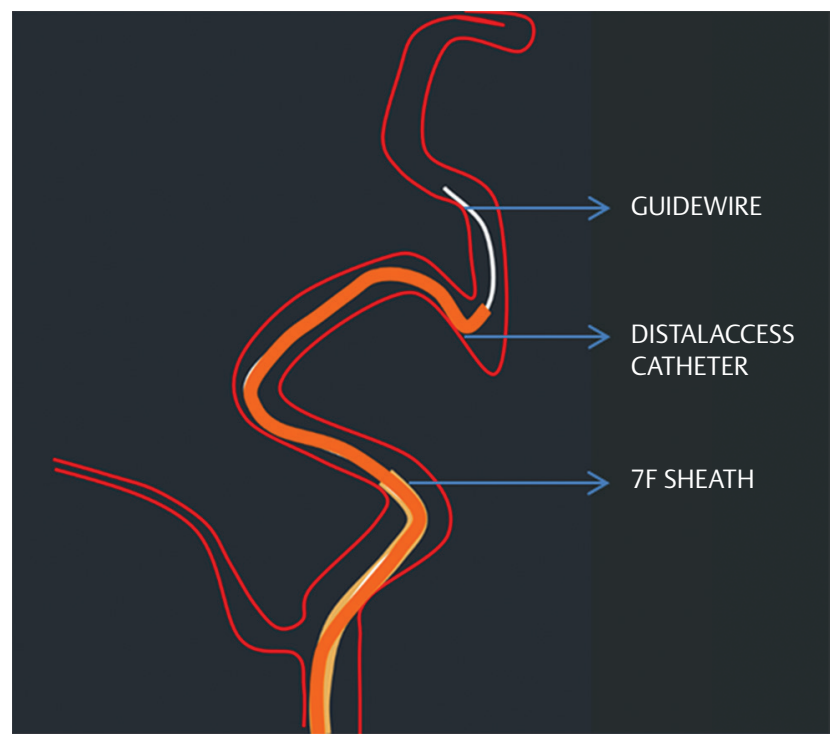

Fig. 5 Taking a distal access catheter through a long sheath placed in the distal CCA can help overcome problems in the internal carotid artery.

is a very high risk of the sheath slipping out of the skin during the procedure with massive hematoma in the neck. The rest of the procedure can be performed through the guiding catheter, which will be now near the groin.

While removing the sheath, all you need is compression for approximately 30 minutes. However, ensure that an ultrasound is done to make sure that there is no pseudoaneurysm. Also, carotid compression is ideal after reversal of heparin.

\section{Crossing a Large Aneurysm into the Distal Vessel}

Crossing into the distal vessel can be a challenge whenever a stent-assisted coiling or a flow diverter is to be placed across the neck of a large aneurysm. To achieve this, the wire is allowed to loop inside the aneurysm and then go distally (-Fig. 7). The microcatheter is then tracked over the wire, and this combination is taken to a point as distal as possible. Once this is achieved, the wire is removed and a blank road map taken. Then, the microcatheter is filled with contrast for visibility. Now, the loop inside the aneurysm can be straightened by gently pulling on the catheter until the loop is completely straightened (-Fig. 8). Thus, it is important that the microcatheter tip is as distal as possible so that it does not fall back into the aneurysm sac. ${ }^{6}$

\section{Conclusion}

It is often said that difficult vascular anatomy can pose a challenge to even a most experienced interventional radiologist. However, a combination of good techniques, proper wires, long sheaths, guiding catheters, and other similar hardware can help us overcome this problem. It is also prudent to remember that at times when nothing works, it would be wiser to stop the procedure and think of an alternative way to treat the patient. 


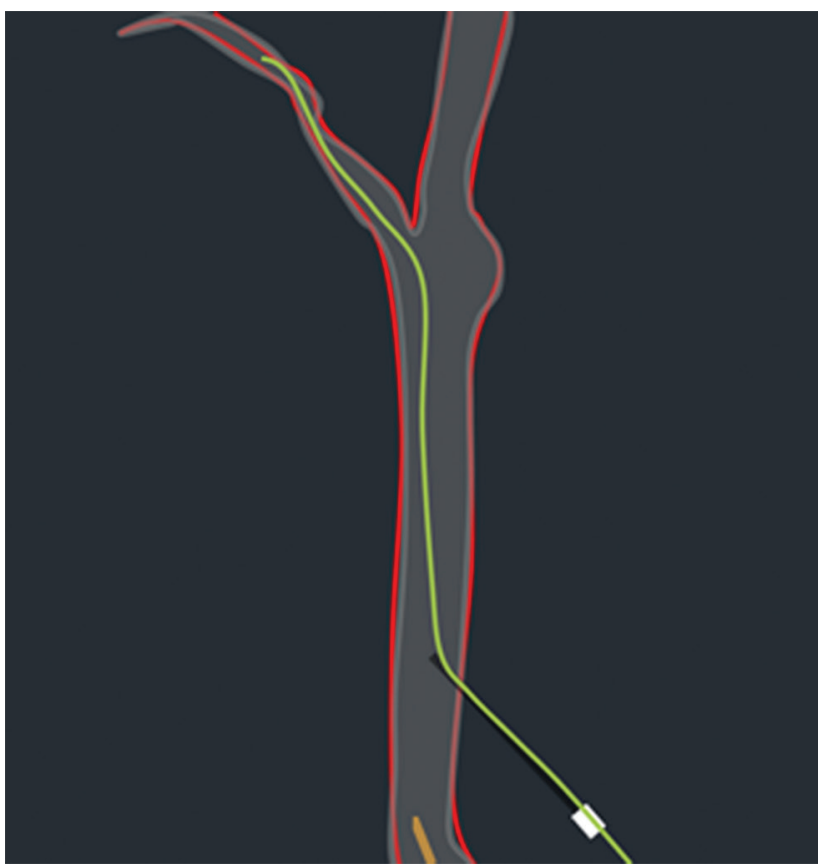

Fig. 6 Accessing the carotid artery directly is possible by using a pediatric puncture set and with a guidewire navigated into the external carotid artery.

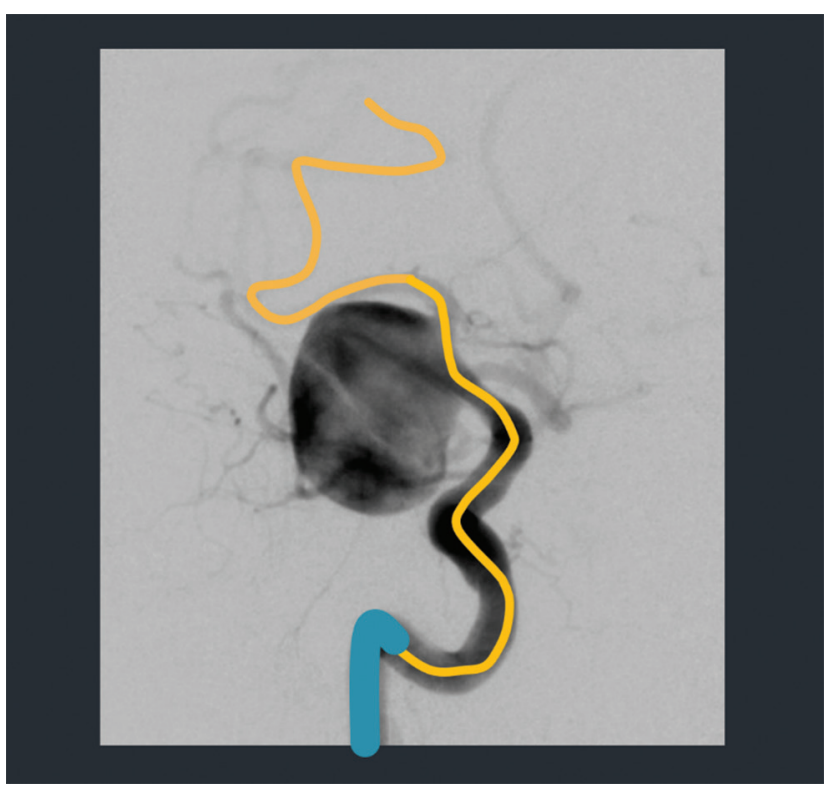

Fig. 8 The wire is withdrawn, and then the catheter is generally pulled until the loop is straightened.

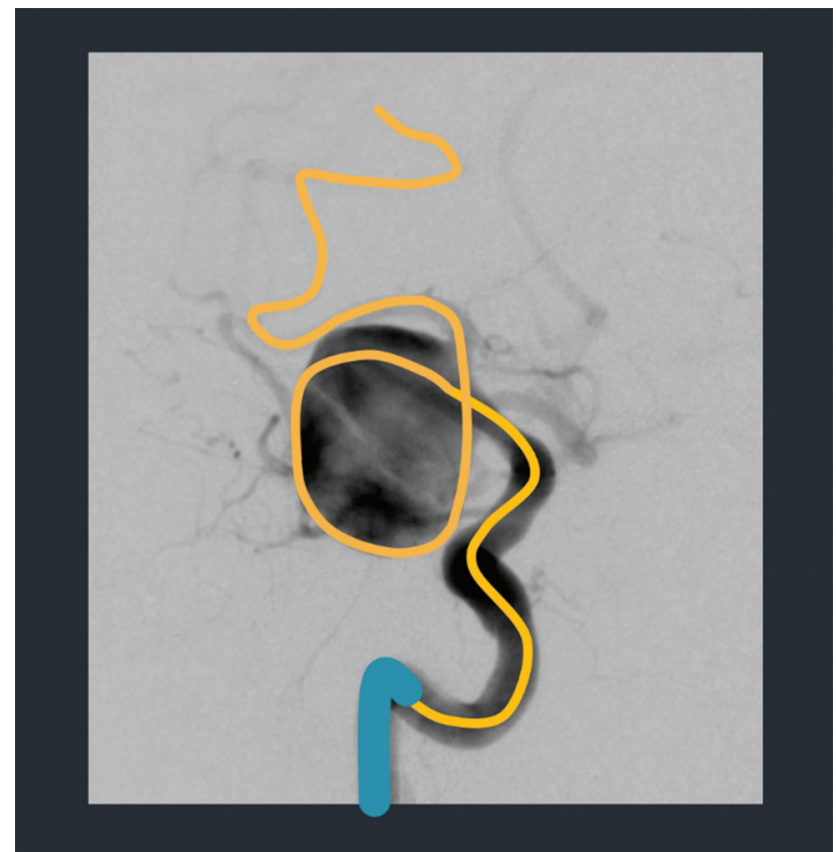

Fig. 7 A loop is created with the wire and the microcatheter navigated over it.

\section{References}

1 Nishino K, Ito Y, Hasegawa H, Kikuchi B, Fujii Y, Tanaka R. Modified buddy wire technique for coil embolization of posterior circulation aneurysms. Neuroradiology 2007;49(1):49-55

2 Singh J, Wolfe SQ Janjua RM, Hedayat H, Burnette C. Anchor technique: use of stent retrievers as an anchor to advance thrombectomy catheters in internal carotid artery occlusions. Interv Neuroradiol 2015;21(6):707-709

3 Vanzin JR, Bambini Manzato L, Slaviero F, Strzelecki M, D’agostini Annes R. Direct vertebral artery access for the endovascular treatment of basilar artery aneurysms. Interv Neuroradiol 2012;18(1):29-32

4 Berman HL, Cornell TJ. A technique for reforming the Simmons curved cerebral catheters. AJR Am J Roentgenol 1982;139(4):824-824

5 Bauer AM, Rasmussen PA. Treatment of intracranial vasospasm following subarachnoid hemorrhage. Front Neurol 2014;5:72

6 Kan P, Wakhloo AK, Mokin M, Puri A. Techniques in distal access of wide-necked giant intracranial aneurysms during treatment with flow diversion. Surg Neurol Int 2015;6(8, Suppl 7):S284-S288 